\title{
ARTICLE OPEN \\ Helicobacter suis infection alters glycosylation and decreases the pathogen growth inhibiting effect and binding avidity of gastric mucins
}

\author{
Médea Padra ${ }^{1}$, Barbara Adamczyk ${ }^{1,2}$, Bram Flahou ${ }^{3}$, Mattias Erhardsson ${ }^{1}$, Gurdeep Chahal ${ }^{1}$, Annemieke Smet ${ }^{3,4}$, Chunsheng Jin ${ }^{1}$, \\ Anders Thorell ${ }^{5}$, Richard Ducatelle ${ }^{3}$, Freddy Haesebrouck ${ }^{3}$, Niclas G. Karlsson ${ }^{1}$ and Sara K. Lindén ${ }^{1}$
}

\begin{abstract}
Helicobacter suis is the most prevalent non-Helicobacter pylori Helicobacter species in the human stomach and is associated with chronic gastritis, peptic ulcer disease, and gastric mucosa-associated lymphoid tissue (MALT) lymphoma. $H$. suis colonizes the gastric mucosa of $60-95 \%$ of pigs at slaughter age, and is associated with chronic gastritis, decreased weight gain, and ulcers. Here, we show that experimental $H$. suis infection changes the mucin composition and glycosylation, decreasing the amount of $H$. suisbinding glycan structures in the pig gastric mucus niche. Similarly, the $H$. suis-binding ability of mucins from $H$. pylori-infected humans is lower than that of noninfected individuals. Furthermore, the $H$. suis growth-inhibiting effect of mucins from both noninfected humans and pigs is replaced by a growth-enhancing effect by mucins from infected individuals/pigs. Thus, Helicobacter spp. infections impair the mucus barrier by decreasing the $H$. suis-binding ability of the mucins and by decreasing the antiprolific activity that mucins can have on $\mathrm{H}$. suis. Inhibition of these mucus-based defenses creates a more stable and inhabitable niche for $H$. suis. This is likely of importance for long-term colonization and outcome of infection, and reversing these impairments may have therapeutic benefits.
\end{abstract}

Mucosal Immunology (2019) 12:784-794; https://doi.org/10.1038/s41385-019-0154-4

\section{INTRODUCTION}

Helicobacter suis is a worldwide spread Gram negative, motile, spiral-shaped bacterium, which colonizes the gastric mucosa of $60-95 \%$ of pigs at slaughter age. ${ }^{1-3} H$. suis infection is associated with chronic gastritis, decreased daily weight gain, ${ }^{4}$ and the development of ulcers in the pars oesophagea of the pig stomach. ${ }^{1,3,5}$ In the human stomach, $H$. suis is the most prevalent non-Helicobacter pylori Helicobacter (NHPH) species. ${ }^{6} \mathrm{H}$. suis infection in the human stomach has been associated with the development of chronic gastritis, ${ }^{7}$ peptic ulcer disease, ${ }^{8}$ and gastric mucosa-associated lymphoid tissue (MALT) lymphoma. ${ }^{9}$ Although the prevalence of $\mathrm{NHPH}$ infection in humans is low (between $0.2 \%$ and $6 \%,{ }^{10,11}$ ) the risk of developing gastric MALT lymphoma is higher during NHPH infection compared with H. pylori infection. ${ }^{12,13}$

The mucus layer is the first barrier gastric pathogens must overcome for colonization. The main components of the mucus layer are the densely glycosylated mucin glycoproteins. ${ }^{14,15}$ In the healthy human stomach, MUC5AC and MUC6 are the main secreted mucins, but during disease, MUC2 and MUC5B can also appear. ${ }^{16,17}$ Proteomic analysis of mucins from healthy pigs identified predominantly MUC5AC, but MUC6 and MUC5B might also be members of the pig gastric mucin repertoire. ${ }^{18}$
The high diversity of mucin $O$-glycans provides an extensive repertoire of bacterial attachment sites and O-glycans take part in host-pathogen interactions in the gastrointestinal tract. ${ }^{19-22}$ Mucus can be beneficial for bacteria by providing nutrients for growth ${ }^{23,24}$ and protecting bacteria from the harmful environment. $^{25}$ Mucins can also prevent close contact of the pathogen with the gastric epithelial cells by binding, which may contribute to removing the bacteria from their niche with the gastric emptying. ${ }^{26-28}$ Binding to mucin glycans can also inhibit bacterial growth and affect expression of virulence genes. ${ }^{29,30}$

The mucin glycan structures show high variation between species, individuals, and tissue locations within each individual, ${ }^{19,27,31-33}$ and their production can be affected by bacterial colonization. ${ }^{31,33}$ For example, infection with $H$. pylori has been demonstrated to alter the expression, turnover rate, and glycosylation of host gastric mucins. ${ }^{26,34,35}$ Among mucins isolated from the stomach of healthy pigs, mass spectrometric analysis identified 109 O-glycan structures with high inter-individual diversity. ${ }^{18} \mathrm{H}$. suis binding to pig gastric mucins and glycolipids has been demonstrated, and two mucin-binding modes of this bacterium have been revealed: binding to structures terminating with galactose at both neutral and acidic $\mathrm{pH}$ and to negatively charged structures at acidic $\mathrm{pH}^{18}$

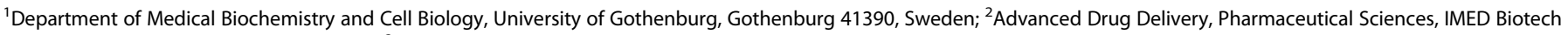

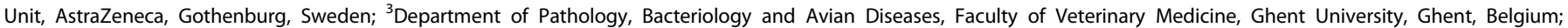

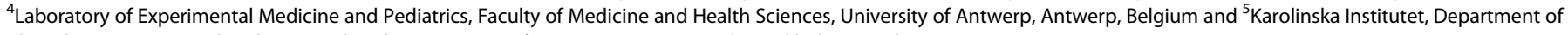
Clinical Science at Danderyds Hospital and Department of Surgery, Ersta Hospital, Stockholm, Sweden

Correspondence: Sara K. Lindén (sara.linden@gu.se)

Senior authors: Freddy Haesebrouck, Niclas G. Karlsson, Sara K. Lindén
}

Received: 27 September 2018 Revised: 11 January 2019 Accepted: 10 February 2019

Published online: 7 March 2019 
The aim of the present study was to investigate the effect of infection-related changes on $\mathrm{H}$. suis interactions with mucins. We studied the effect of experimental $H$. suis infection on pig gastric mucin glycosylation as well as downstream effects of these infection-induced changes on $\mathrm{H}$. suis binding and growth. Finally, we examined $H$. suis binding and growth in response to mucins isolated from human stomachs with and without $H$. pylori infection.

\section{RESULTS}

Histological analysis of pig gastric tissue sections after experimental inoculation with $H$. suis

Fluorescent in situ hybridization (FISH) revealed $H$. suis in the mucus layer overlying the epithelial surface (Fig. 1e) and throughout the entire length of the pig gastric pits (Fig. 1f), 4 weeks post inoculation. Signs of inflammation were detected in tissue sections from three out of four infected pigs. The severity of inflammation varied between pigs: no histological signs of inflammation were detected in one pig, whereas in the others the signs ranged from mild inflammation with inflammatory cell infiltration to severe inflammation with markedly enlarged lymphoid follicles (Figs. 1g, 2a). No signs of inflammation were detected in the sham-inoculated noninfected pig gastric tissue sections (Fig. 1c). The mucusproducing cells lining the surface and gastric pits produced both neutral and acidic mucins in both the infected and noninfected group. We observed a shift in color of mucins from magenta to purple among $H$. suis-infected Alcian blue/periodic acid-Schiff stained tissue sections, which reflects an increased level of acidity of mucin glycans after infection (Fig. 1d, h).

$H$. suis infection causes decreased MUC5AC levels Mucins were isolated from the antrum of $H$. suis-infected $(n=4)$ and noninfected ( $n=3)$ pigs, and the mucin-containing fractions were pooled to provide four samples (surface soluble (SS), surface insoluble $(\mathrm{SI})$, gland soluble $(\mathrm{Gl})$, and gland insoluble (GI)) from each individual for further experiments. The purified mucins were analyzed for density, glycan, and MUC5AC content. Quantification of mucins based on their carbohydrate content revealed that pig gastric mucins were predominantly insoluble in guanidinium chloride $(\mathrm{GuHCl})$, both in the noninfected $(60 \%)$ and $H$. suis-infected (63\%) groups (Fig. 2b). No difference in

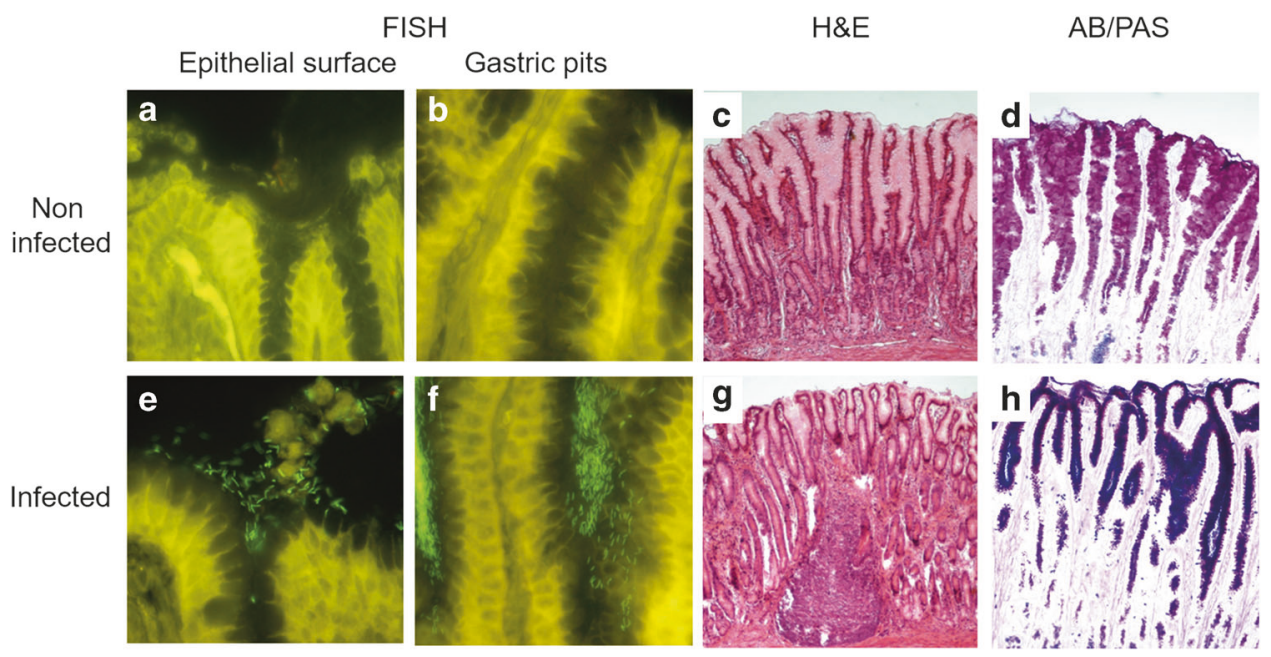

Fig. 1 Representative images of pig gastric tissue sections with or without $H$. suis infection. $\mathbf{a}, \mathbf{b}$, e and $\mathbf{f}$ Presence and spatial distribution of $H$. suis determined by fluorescent in situ hybridization (FISH). $\mathbf{a}, \mathbf{b}$ Absence of $H$. suis in sections from noninfected pigs. e, $\mathbf{f}$ Presence of $H$. suis (bright green) in the surface mucus layer and gastric pits of tissue sections from $H$. suis-infected pigs. c Hematoxylin and eosin (H\&E)-stained tissue section from a noninfected pig. $\mathbf{g ~ H}$ HE-stained section from an $\mathrm{H}$. suis-infected pig showing presence of a lymphoid follicle. $\mathbf{d}$ AB/PASstained section of a noninfected pig. $\mathbf{h}$ AB/PAS-stained section of an $H$. suis-infected pig. The AB/PAS stains neutral glycans pink, acidic mucin glycans blue, and where overlap occurs, the color is magenta

a

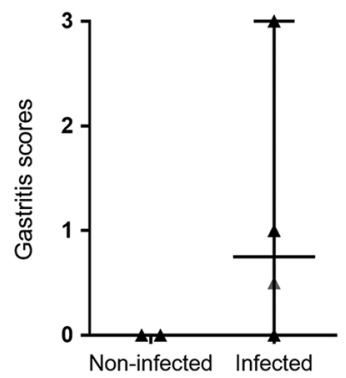

b

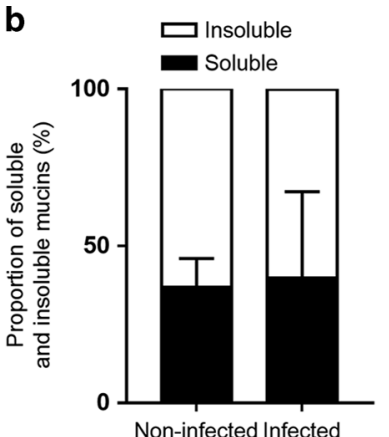

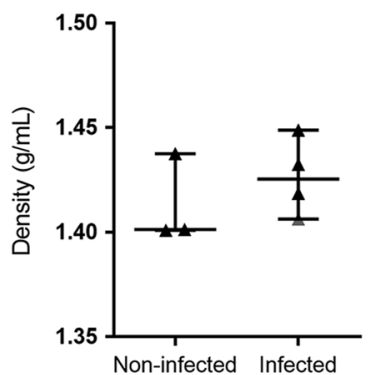

d

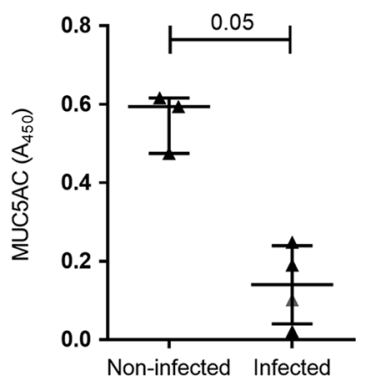

Fig. 2 Gastritis scores and characteristics of purified pig gastric mucins. a Gastritis scores from experimentally infected and sham-infected pigs. b Proportion of pig gastric mucins that were soluble versus insoluble in guanidinium chloride (GuHCl). The insoluble mucins were solubilized by reduction and alkylation. $c$ The density $(\mathrm{g} / \mathrm{mL})$ of pig gastric mucins was similar among noninfected and infected pigs $(p>0.05)$. d The MUC5AC level was lower among gastric-soluble mucins from $H$. suis-infected pigs than from noninfected ones. The MUC5AC signal was normalized for the glycan content of each sample, and the median of the four mucin sources for each pig is presented in the graph ( $p=0.05$ ). Statistics: Mann-Whitney $U$ test. The pig marked in gray carried a second $H$. suis strain in addition to the strain used in the experimental infection 
Table 1. Apomucins in pig gastric mucin samples detected by proteomic analysis

\begin{tabular}{|c|c|c|c|c|c|}
\hline \multirow[t]{2}{*}{ Sample } & \multicolumn{4}{|c|}{ Apomucin } & \multirow[t]{2}{*}{ Infection status } \\
\hline & MUC5AC & MUC5B & MUC6 & MUC2 & \\
\hline Pig1 SS & +++ & + & & & Noninfected \\
\hline Pig1 SI & +++ & + & & & \\
\hline \multicolumn{6}{|l|}{ Pig1 GS } \\
\hline Pig1 GI & ++ & + & + & & \\
\hline \multicolumn{6}{|l|}{ Pig2 GS } \\
\hline Pig2 SI & +++ & ++ & + & & \\
\hline Pig2 GI & + & + & & & \\
\hline Pig4 SS & + & & + & & Infected \\
\hline Pig4 SI & + & + & & & \\
\hline Pig4 GS & & & +++ & & \\
\hline Pig5 SI & + & + & & & \\
\hline Pig5 GS & + & + & + & & \\
\hline Pig5 Gl & ++ & + & & & \\
\hline Pig7 SS & + & & & & \\
\hline Pig7 SI & ++ & + & & & \\
\hline Pig7 GS & & & + & & \\
\hline Pig7 GI & ++ & + & & + & \\
\hline
\end{tabular}

Proteomic tandem mass spectrometry analysis was carried out on surface soluble (SS), surface insoluble (SI), gland soluble (GI), and gland insoluble (GI) gastric mucins from $H$. suis-infected and noninfected pigs. The mucin content was graded from + to +++ , based on the sum of all Mascot scores with which a particular mucin was found in the experiment $(+$ protein score $1-800,++$ protein score $800-1600,+++$ protein score $1600-2400$ )

mucin density was observed between noninfected and infected pigs ( $p>0.05$, Fig. 2c). Mass spectrometry analysis identified porcine MUC5AC in all samples originating from the surface and in some samples originating from the glands (Table 1). The analysis indicated lower levels of MUC5AC among mucins from infected pigs than from noninfected pigs and a decrease in MUC5AC content in the mucin samples derived from $H$. suisinfected pig stomachs compared with the samples from the noninfected group was confirmed using ELISA (Fig. $2 \mathrm{~d}, p=0.05$ ). MUC5B and MUC6 were also identified in some samples (Table 1).

\section{$H$. suis infection changes pig gastric mucin glycosylation}

Mucin O-glycans from $H$. suis-infected $(n=4)$ and noninfected $(n=3)$ pigs were characterized by mass spectrometry. Of the 118 different oligosaccharides that were found (Fig. 3a, Supplementary Table 1), 93 were present in both groups, 18 were uniquely detected among the noninfected group, and 7 were only detected in the infected group (Fig. 3b). Unique structures in controls contained 0-1 fucose residues and only low amounts of sialylation ( 2 structures were monosialylated), while unique structures in infected group contained up to 2 fucose and/or sialic acids (Supplementary Table 2). The median (IQR) number of different glycan structures detected in the gastric mucin samples was 65 (47-75) in the noninfected group and 45 (36-65) in the infected group, implying a decrease in the number of glycan structures on pig gastric mucins upon infection $(p \leq 0.05$, Fig. 3c). H. suis has been shown to bind to acidic (sialylated and/ or sulphated) glycan structures and glycans terminating with galactose (including structures terminating with type 1 $\mathrm{N}$-acetyllactosamine, Gal $\beta 1,3 \mathrm{GICNAC}) .{ }^{18}$ Here, we found an increase in the relative abundance of acidic (mostly sulfated) glycan structures $(p<0.05$, Fig. $3 d$ ) and a decrease in glycan structures terminating with galactose, including the Gal $\beta 1,3 \mathrm{Glc}$ NAc epitope, in the infected pig gastric mucin samples compared with the noninfected samples $(p<0.01$, Fig. $3 d)$. In addition, fucosylation (identified as blood group $A$ and $H$ epitopes) was also increased in the infected mucin group ( $p<$ 0.05, Fig. 3d).

Decreased $H$. suis-binding ability of mucins isolated from infected pigs and humans

$H$. suis bound to all four types of mucins (surface soluble, surface insoluble, gland soluble, and gland insoluble) isolated from both noninfected and infected pigs. The level of $H$. suis binding to mucins differed between pigs and the four different mucin sources within each pig, as could be expected based on differences in glycosylation. In line with previous results, ${ }^{18}$ the binding avidity of $H$. suis to pig gastric mucins was sevenfold higher at $\mathrm{pH} 2$ than at neutral $\mathrm{pH}$ (Fig. 4). At $\mathrm{pH} \mathrm{7,} \mathrm{H.} \mathrm{suis} \mathrm{binding}$ was lower to the mucin samples isolated from infected pigs $(p<$ $0.05)$, whereas at $\mathrm{pH} 2$ there was only a trend in the same direction $(p>0.05)$, matching the changes in $H$. suis-binding structures (Fig. 3d). Paralleling these results, the level of binding correlated with the $H$. suis density in tissue sections at $\mathrm{pH} 7(p=0.02, \mathrm{r}=$ $-0.92)$, but not at $\mathrm{pH} 2(p=0.51, r=-0.3)$. When comparing mucin binding on a pig level, calculating the mean binding of the four mucin sources for each pig, the decreased binding ability of the infected pigs was not statistically significant, although the trend remained (Fig. 4d). The lack of statistical significance is likely due to the low number of noninfected pig samples available. Since we had analyzed over 70 pigs to obtain three $H$. suis-free pigs, we decided to investigate the effect of infection among human samples instead of attempting to expand the number of pigs further.

To study the effect of Helicobacter spp. infection on binding of $H$. suis to human gastric mucins, binding assays were carried out using purified human gastric mucins isolated from the antral part of the stomach of Helicobacter spp. infected $(n=6)$ and noninfected $(n=7)$ individuals. All samples that were Helicobacter spp. positive by PCR using Helicobacter genus-specific PCR primers were also positive using three $H$. pylori-specific primer pairs, suggesting that $100 \%$ of the Helicobacter spp. positive samples in the patient group were infected with $H$. pylori. Unlike pig mucins, human gastric mucins had similar $H$. suis-binding avidity at $\mathrm{pH} 2$ and at $\mathrm{pH} 7$ (Fig. 5). Gastric mucins from $\mathrm{H}$. pyloriinfected individuals had decreased $H$. suis-binding ability compared with those from noninfected individuals, at both neutral and acidic $\mathrm{pH}(p<0.01$, Fig. 5). The difference in amplitude between noninfected and infected mucins was similar for pig and human samples, but the larger number of human samples rendered the difference statistically significant on an individual level too (Fig. 5b, d).

Mucins from infected pigs and humans increase $H$. suis growth To study the growth of $H$. suis in response to mucins, we cultured $H$. suis in the presence of gastric mucins isolated from infected and noninfected individuals. $H$. suis growth decreased by $4 \%$ when cultured in the presence of gastric mucins from noninfected pigs and increased by $12 \%$ in the presence of mucins from infected pigs, compared with $H$. suis cultured without mucins (Fig. 6a, b, $p \leq 0.05)$. Similar results were obtained after culture with the human mucins: a $4 \%$ decrease in growth was observed with mucins from noninfected stomachs and a $4 \%$ increase in the presence of mucins from $\mathrm{H}$. pylori-infected individuals (Fig. $6 c, p<$ 0.05).

H. suis growth increases in response to fucose and decreases from galactose

Mucins can promote or inhibit bacterial growth depending on the glycan structures they carry. ${ }^{29}$ The amount of a1,4GICNAc, a 
a

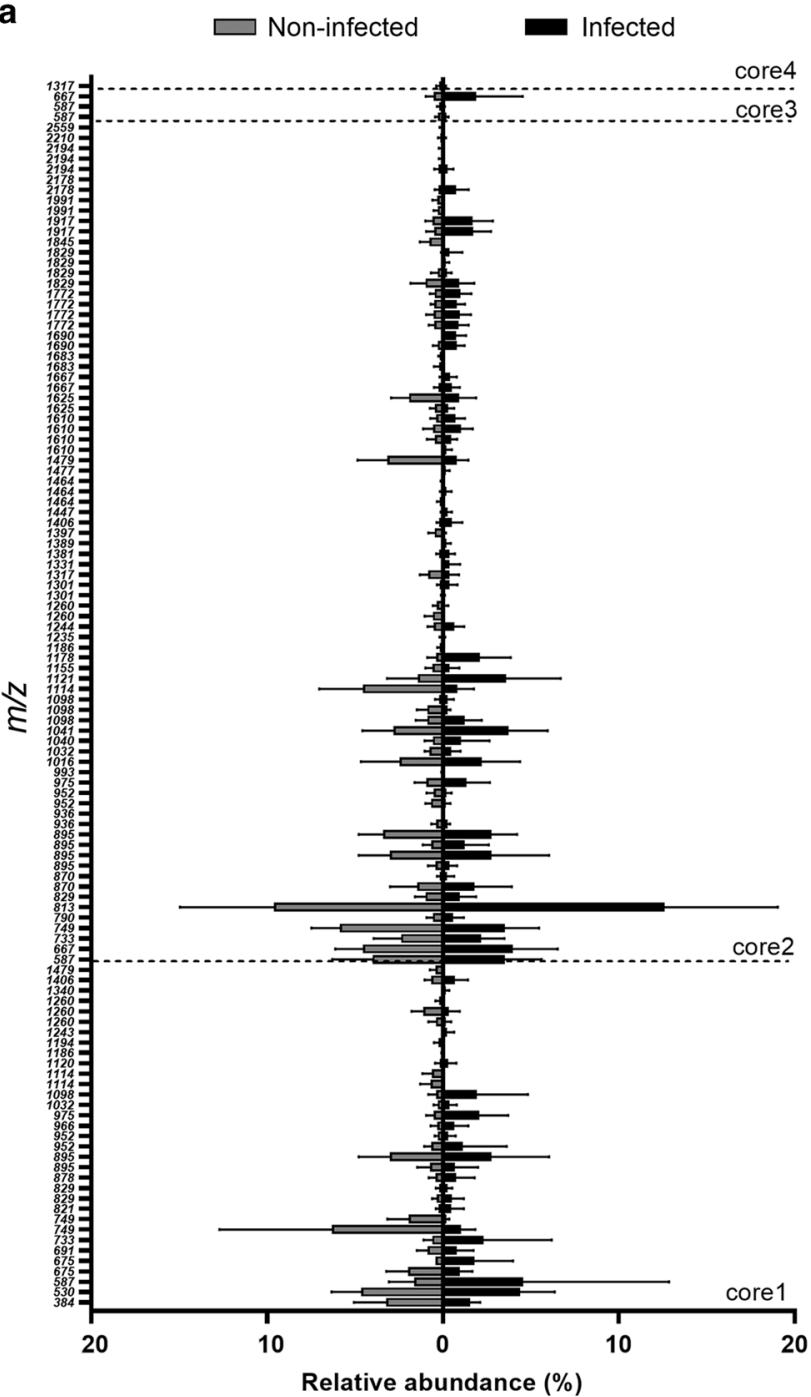

b

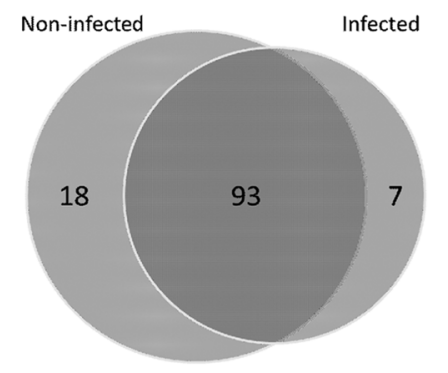

C

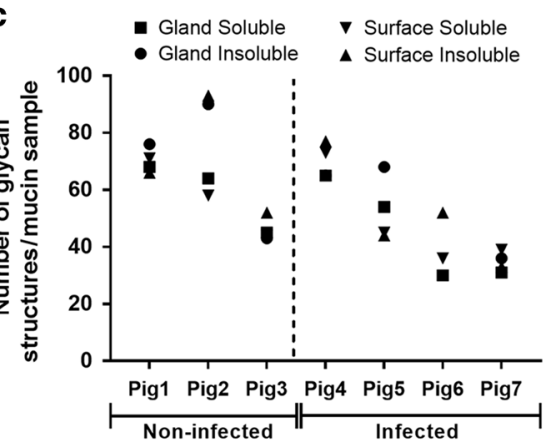

d

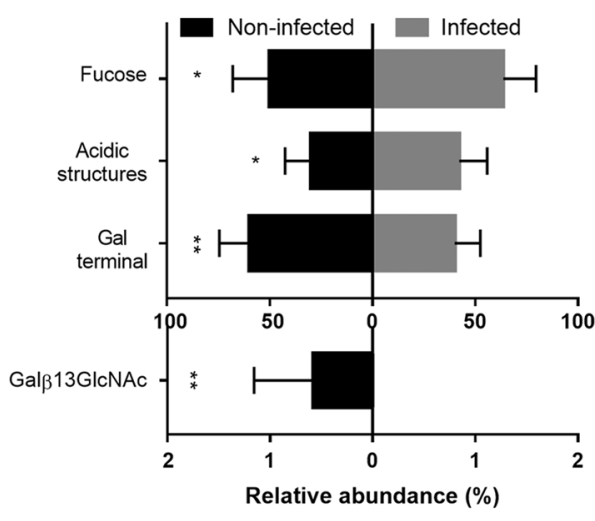

Fig. 3 Glycosylation of gastric mucins from $H$. suis-infected and noninfected pigs. a Relative abundance (\%) of each of the identified glycan structures among $H$. suis-infected and noninfected pig gastric mucin samples. The full list of identified glycans is available in Supplementary Table 1. b Distribution of common and unique mucin glycan structures in noninfected and infected pig groups. c Number of glycan structures identified in individual gastric mucin samples isolated from noninfected and infected pigs. $\mathbf{d} H$. suis infection-associated alterations in the relative abundance of terminal epitopes on pig gastric mucins. Many of the structures are branched, leading to that the sum of the terminal structures is above $100 \%$. The relative abundance of fucosylated and acidic glycan structures increases, whereas that of the galactoseterminating glycan structures (including Gal $\beta 1,3 \mathrm{GlcNAC}$ ) decreases with infection. Statistics: ${ }^{*} p<0.05$, ${ }^{* *} p<0.01$, two-way ANOVA, data are plotted as mean \pm SEM

structure previously suggested to act as a natural antibiotic against $H$. pylori, ${ }^{36}$ was similar among noninfected and infected pigs $(11.7 \pm 3.1$ vs. $6.6 \pm 1.15 \%, p>0.05)$, which suggests that this structure is not responsible for the difference in $H$. suis growth response. We analyzed the relation between the effect of the pig gastric mucins on $H$. suis growth, and the relative abundance of glycan structures identified on these mucins. We observed a positive correlation between $H$. suis growth and the relative abundance of fucosylated ( $r=0.5501, p<0.01$, Fig. 7a) as well as acidic ( $r=0.5485, p<0.01$, Fig. $7 b)$ glycan structures, whereas the relative abundance of mucin glycans terminating with galactose (that did not carry fucose and/or acidic glycans) negatively correlated with $H$. suis growth ( $r=0.6844, p<0.001$, Fig. 7c). No correlation was identified with any other terminally located residues.

To verify the effect of fucose, galactose, and acidic structures (sialylated and/or sulfated) on $H$. suis growth, the bacteria were cultured with fucose, galactose, and NeuAc monosaccharides. GIcNAc, the abundance of which did not correlate with the growth of $H$. suis, was also included in the assay. In line with the correlations, fucose promoted $(p<0.05)$ and galactose inhibited $H$. suis growth $(p<0.01)$, whereas GlcNAc had no effect $(p>0.05)$, compared with $H$. suis growth in the absence of added monosaccharides (Fig. 8). NeuAc did not affect $H$. suis growth ( $p$ $>0.05$ ), which suggests that instead sulfation may stimulate growth (sulfation was also more abundant), although the association with acidic glycans might also not be causative.

\section{DISCUSSION}

In the present study, we showed that experimental $H$. suis infection changed the mucin composition and glycosylation, which decreased the amount of $H$. suis binding glycan structures in the pig gastric mucus niche. Similarly, the $H$. suis-binding and 

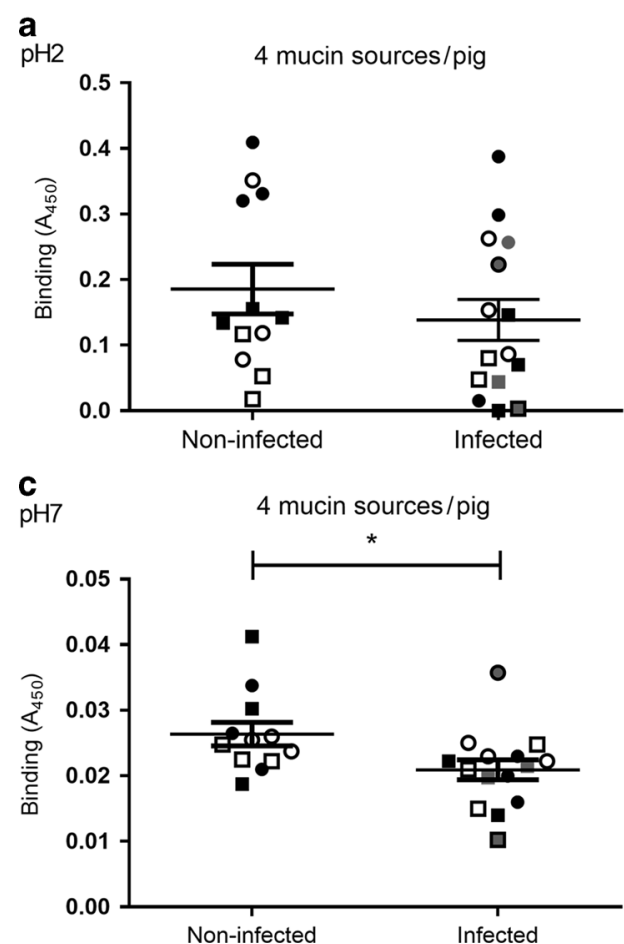

b

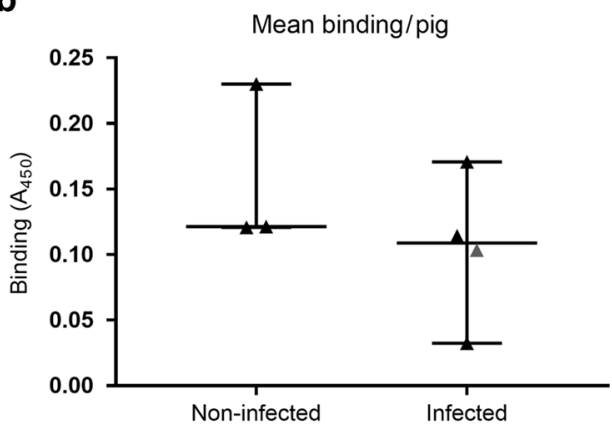

d

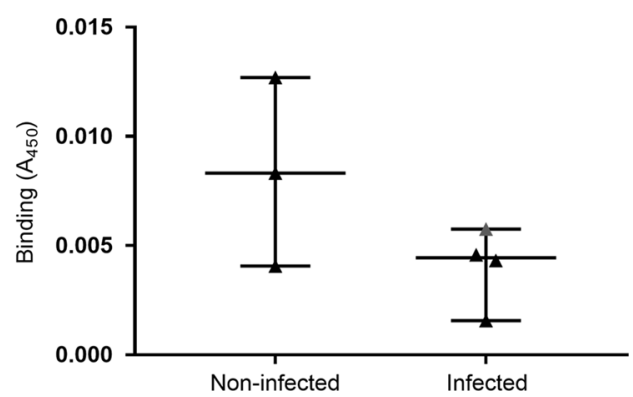

Fig. $4 \quad H$. suis binding to pig gastric mucins isolated from pigs with or without $H$. suis infection. $\mathbf{a}, \mathbf{b} H$. suis binding to pig gastric mucin samples at $\mathrm{pH}$ 2. a The data points represent the binding to surface soluble, surface insoluble, gland soluble, and gland insoluble mucins from each pig. b The data points represent the average binding signal from the four mucin sources from each pig. $\mathbf{c}$, d $H$. suis binding to pig gastric mucin samples at $\mathrm{pH}$ 7. c The data points represent the binding to surface soluble, surface insoluble, gland soluble, and gland insoluble mucins from each pig. $\mathbf{d}$ The data points represent the average binding signal from the four mucin sources from each pig. $\bigcirc$ Surface soluble, - Gland soluble, $\square$ Surface insoluble, $\square$ Gland insoluble, $\Delta$ Mean binding value of the four mucin sources per pig. The pig marked in gray carried a second $H$. suis strain in addition to the strain used in the experimental infection. Statistics: a, c: ${ }^{*} p<0.05$, two-way ANOVA, the bars represent mean $\pm S E M, \mathbf{b}, \mathbf{d}$, the bars represent median with range. The results were reproduced twice

growth-inhibiting ability of gastric mucins from $H$. pylori-infected human stomachs was lower than those from noninfected individuals.

After experimental infection, we detected $H$. suis in the mucus layer covering the gastric epithelial surface as well as in the entire length of gastric pits. Experimental $H$. suis infection decreased the amount of MUC5AC in the tissue, in line with previous observations in $H$. pylori-infected human gastric tissues. ${ }^{37}$ Among purified pig gastric mucins, we identified 118 different oligosaccharides, the number and composition of which structures differed between infected and noninfected pigs. Fewer glycan structures were identified among infected pigs compared with uninfected pigs, in spite of fewer pigs in the latter group. This is in contrast to observations on pig colonic mucins, where Brachyspira hyodysenteriae infection led to production of an increased array of glycan structures. ${ }^{33}$ The disparate glycan responses could be due to differences between the infecting pathogens, host immunological responses, or body locations.

In addition to the experimental pig infection, we also analyzed $H$. suis interactions with human gastric mucins isolated from $H$. pylori-infected and noninfected individuals. Due to the low prevalence of $H$. suis, and limited number of people that go through gastrectomy, it was not feasible to collect a sufficient number of stomachs from $H$. suis-infected humans to do the analysis on specifically $H$. suis-infected human specimens. However, the similar effects on both $H$. suis binding and growth of mucins from pigs experimentally infected with $H$. suis and humans naturally infected by $H$. pylori compared with mucins from their noninfected counterparts suggests that infection by these pathogens lead to similar changes regardless of species.
We have previously demonstrated that $H$. suis binding occurs via two modes of adhesion: (1) to galactose-terminating structures at both neutral and acidic $\mathrm{pH}$ and (2) to negatively charged structures at acidic $\mathrm{pH}^{18}$ In contrast to the large variation in mucin-binding specificity and avidity present among $H$. pylori strains, ${ }^{38}$ binding of four $H$. suis strains isolated from primates and pigs to pig and human mucins was very similar, ${ }^{18}$ suggesting low inter-individual variation in binding specificity among $H$. suis strains. Here, we showed that the binding level of $H$. suis to pig gastric mucins was around sevenfold higher at $\mathrm{pH} 2$ than at $\mathrm{pH} 7$, regardless of the infection status of the pigs. At $\mathrm{pH} 7$, we observed decreased binding of mucins isolated from the infected pigs to $H$. suis, which can be explained by the decreased level of galactoseterminating mucin glycans in infected pig mucins providing less potential binding sites for the bacteria. There was no binding difference between the infected and noninfected pig mucins at $\mathrm{pH} 2$, which likely is due to the fact that at acidic $\mathrm{pH}$, adhesion of $H$. suis also occur via a charge-dependent binding mode to acidic glycans. Since the amount of acidic structures increased after infection, that compensates for the loss of adhesion targets caused by the decreased terminal galactose. Human mucins from infected individuals were less efficient at binding to $H$. suis than mucins from noninfected individuals at both $\mathrm{pH} 2$ and $\mathrm{pH} 7$, and the avidity was similar at both pHs. Gastric mucin glycosylation changes affecting pathogen binding have been shown also after infection with $H$. pylori. ${ }^{26} H$. pylori binds to Le ${ }^{b}$ and SLe ${ }^{\mathrm{x}}$ blood group antigens on gastric mucins via blood group antigenbinding adhesin (BabA) and sialic acid-binding adhesin (SabA), respectively, and infection with this pathogen decreases the level of $\mathrm{Le}^{\mathrm{b}}$ and increases the level of sialylation in the gastric mucosa. ${ }^{26,39}$ These glycosylation changes increase $H$. pylori 
a

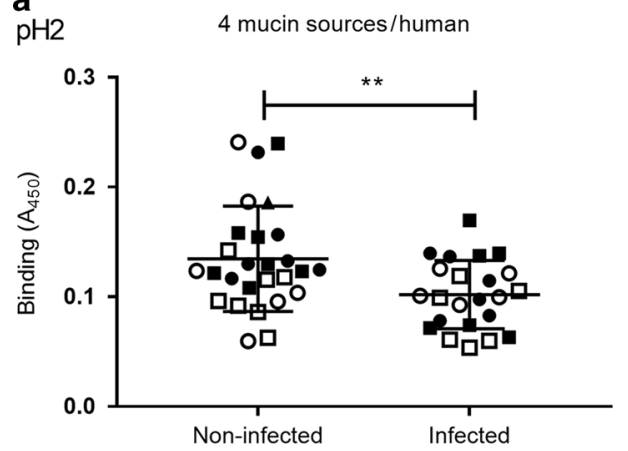

c

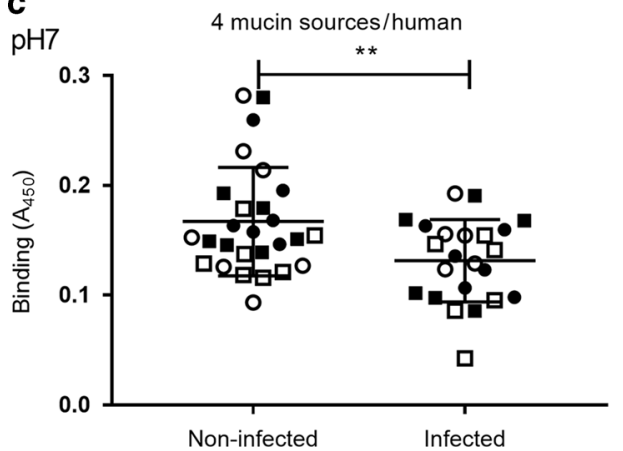

b

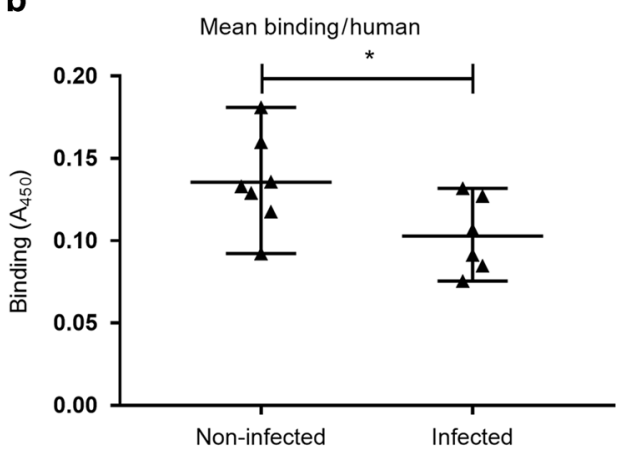

d

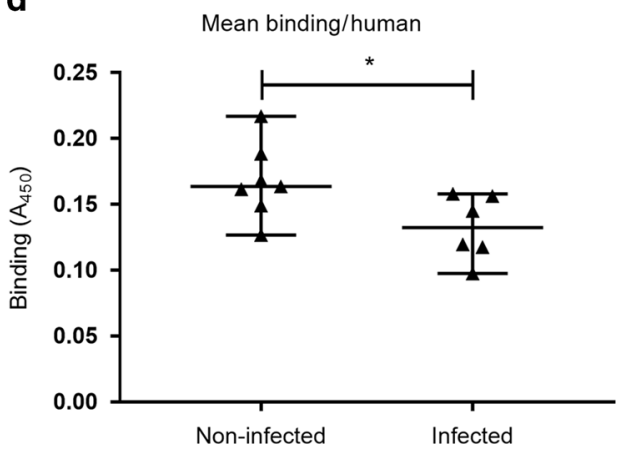

Fig. 5 Binding of $H$. suis to human gastric mucins isolated from individuals with or without $H$. pylori infection. $\mathbf{a}, \mathbf{b} H$. suis binding to human gastric mucin samples at $\mathrm{pH}$ 2. a The data points represent the binding to surface-soluble, surface-insoluble, gland-soluble, and glandinsoluble mucins from each individual. b The data points represent the average binding signal from the four mucin sources from each individual. c, $\mathbf{d} \mathrm{H}$. suis binding to human gastric mucin samples at $\mathrm{pH}$ 7. $\mathbf{c}$ The data points represent the binding to surface soluble, surface insoluble, gland soluble, and gland insoluble mucins from each individual. $\mathbf{d}$ The data points represent the average binding signal from the four mucin sources from each individual. Statistics: $\mathbf{a}, \mathbf{c}$ : ${ }^{* *} p<0.01$, two-way ANOVA, the bars represent mean \pm SEM, $\mathbf{b}$, d: ${ }^{*} p<0.05$, Mann-Whitney $U$ test, the bars represent median with range. O Surface soluble, Gland soluble, $\square$ Surface insoluble, Gland insoluble, $\mathbf{A}$ Mean binding value of the four mucin sources per pig. The pig marked in gray carried a second $H$. suis strain in addition to the strain used in the experimental infection. The graphs are representative of two experiments

binding via SabA and via the charge-dependent binding modes, but decrease binding via BabA to fucosylated structures, including Le $^{\text {b.26,40 }}$ Although the interacting molecules differ between $H$. suis and $H$. pylori, the overall effect of decreased mucin binding ability at neutral $\mathrm{pH}$ after infection is a common feature, as binding avidity via BabA generally is higher than via SabA. ${ }^{30}$

Mucins derived from different individuals and disease states have been shown to have growth promoting or inhibitory effect. $^{29,30}$ For instance, the growth of $H$. pylori can be inhibited by mucins containing a1,4GlcNAc-capped O-glycans, ${ }^{36}$ and $H$. pylori adhesion to mucins can also have growth inhibitory effect by aggregate formation. ${ }^{30}$ Here, we observed that both porcine and human gastric mucins isolated from infected individuals had a growth-promoting effect, whereas mucins from noninfected individuals inhibited the growth of $H$. suis independently of a1,4GlcNAc-capped O-glycan abundance. Instead, we observed a positive correlation between growth and the abundance of acidic and fucosylated structures and a negative correlation with the abundance of galactose terminating structures on the mucins, and culture with fucose and galactose monosaccharides supported these effects. We have previously cultured another bacteria (Aeromonas salmonicida) with twice as high concentration of galactose without inhibition of growth, ${ }^{21}$ suggesting that the inhibition of growth seen in the current study is not affecting all bacteria. Each mucin carried 35-75 glycans, and the growth response to the mucins is thus likely to be the sum of multiple glycans.

$H$. pylori-infected children and rhesus monkeys secreting mucins with less $H$. pylori-binding capacity, develop higher $H$. pylori density and more severe gastritis, ${ }^{26,27}$ supporting the idea that the ability of secreted mucins to bind to $H$. pylori protects the gastric epithelium. Furthermore, mice lacking the Muc1 mucin are more susceptible to infection by $H$. pylori, ${ }^{41}$ and MUC1 acts as a releasable decoy for $H$. pylori binding to the gastric epithelial surface. ${ }^{28}$ Thus, decreased ability of mucins from infected pigs and humans to bind $H$. suis implies that Helicobacter spp. infection inhibits binding and subsequent removal of the pathogen from the gastric niche. Furthermore, increased $H$. suis growth in response to mucins from infected pigs and humans would allow faster growth. In addition, we have previously shown that gastric mucin production is impaired during $H$. pylori infection. ${ }^{35}$ Helicobacter spp. infections thus inhibit three aspects of the mucin-based defense, which in turn creates a more stable and growth-enhancing environment for $\mathrm{H}$. suis to colonize long-term. In addition to creating a more hospitable niche for itself, Helicobacter infection might also lead to increased susceptibility to infection by additional strains or Helicobacter species. Indeed, infection with more than one $H$. pylori strain has been previously reported, ${ }^{42,43}$ and coinfection with $H$. pylori and $\mathrm{NHPH}$ strains has also been described. ${ }^{44,45}$

Although the average differences in growth regulation and binding between mucins from infected versus noninfected individuals were relatively small, the long-term effect in these life-long infections is likely to have a major impact on pathogen density. Furthermore, the difference between the highest and lowest effect of mucins is substantial: for growth, the difference was $35 \%$ after $13 \mathrm{~h}$ and for binding it was 20 -fold. These effects might contribute to the fact that some individuals are asymptomatic carriers whereas others develop severe disease from Helicobacter spp. colonization. 

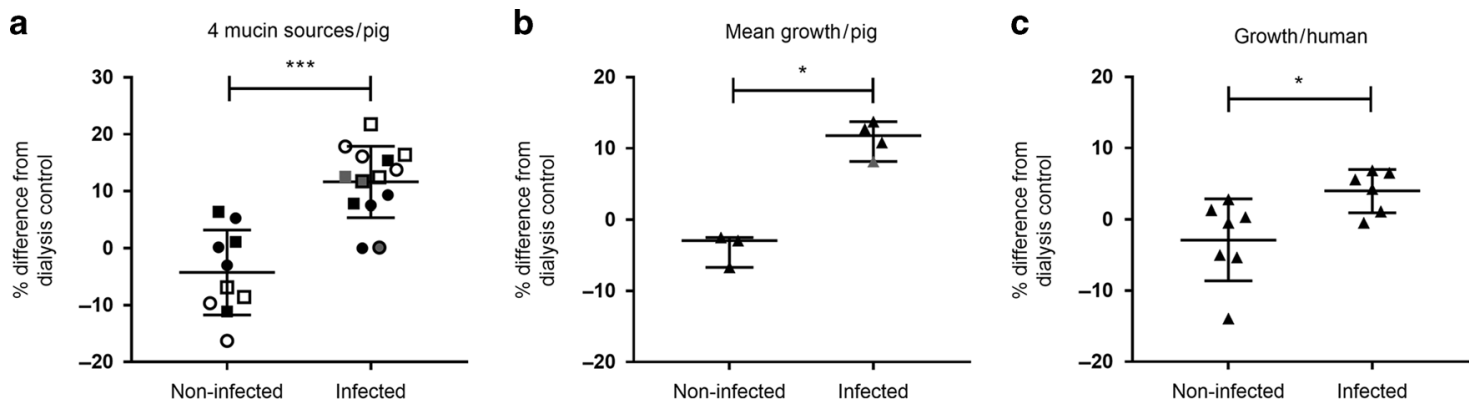

Fig. $6 \mathrm{H}$. suis growth in the presence of gastric mucins from infected or noninfected pigs and humans. $\mathbf{a}$, $\mathbf{b}$ Growth of $H$. suis in the presence of pig gastric mucins isolated from $\mathrm{H}$. suis infected or noninfected pigs. The results are presented in relation to growth in the absence of mucins. a $H$. suis growth in response to four mucin sources per individual (i.e., surface soluble, surface insoluble, gland soluble, and gland insoluble mucins). Statistics: ${ }^{* * *} \mathrm{p}<0.001$, two-way ANOVA, the bars represent mean \pm SEM. b Mean $\mathrm{H}$. suis growth in response to the four mucin sources. The graph shows one data point per individual. Statistics: ${ }^{*} p \leq 0.05$, Mann-Whitney $U$ test, the bars represent median with range. $c H$. suis growth in the presence of human gastric mucins isolated from $\mathrm{H}$. pylori-infected or noninfected individuals. The results are presented in relation to $H$. suis cultured without mucins. Statistics: ${ }^{*} p<0.05$, Mann-Whitney U test, the bars represent median with range. The graph shows one data point per individual. $\bigcirc$ Surface soluble, Gland soluble, $\square$ Surface insoluble, Gland insoluble, $\boldsymbol{\Delta}$ Mean growth in response to the four mucin sources per pig. The pig marked in gray carried a second $H$. suis strain in addition to the strain used in the experimental infection. The graphs are representative of two experiments
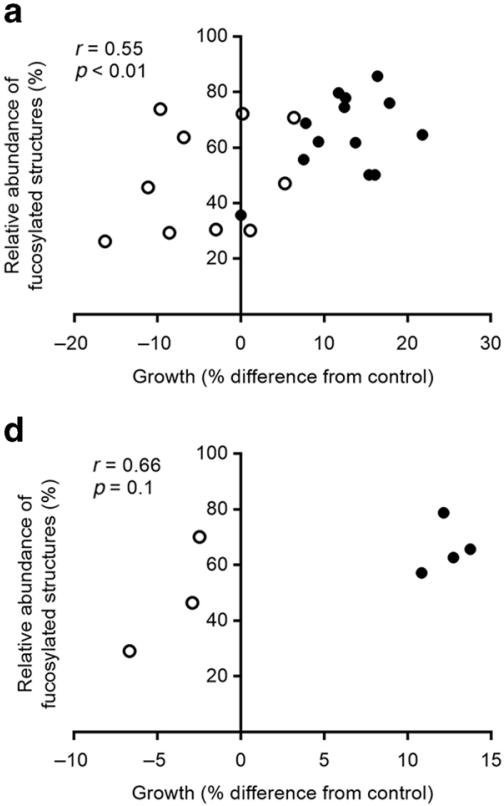

b

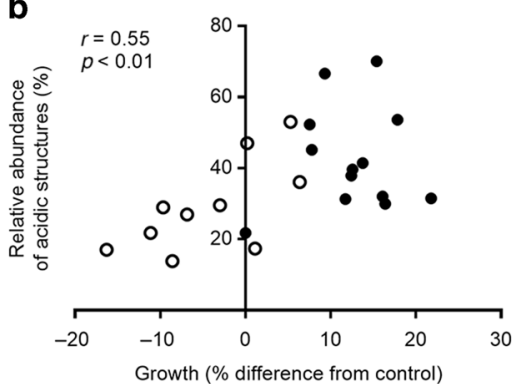

e

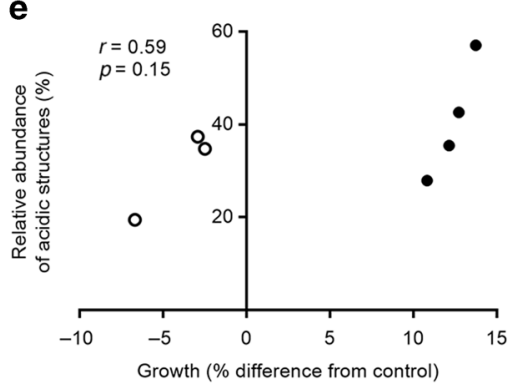

C

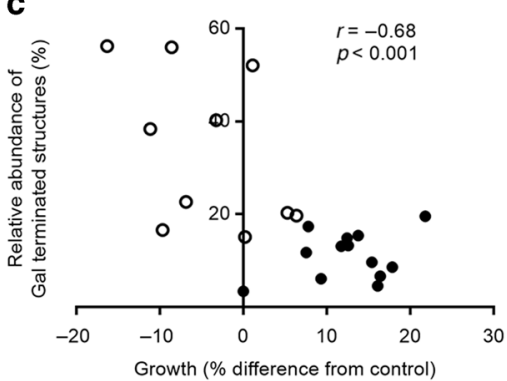

f

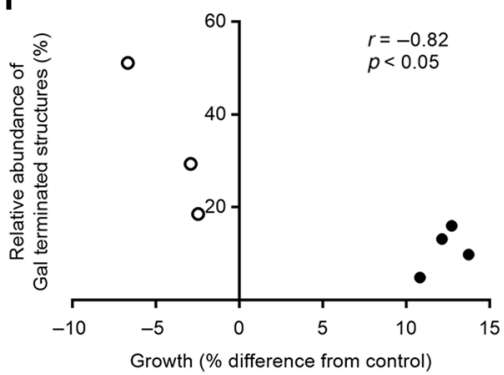

Fig. 7 Relation between the effect of pig gastric mucins on $H$. suis growth and the relative abundance of glycan epitopes carried by these mucins. a, $\mathbf{d}$ Relation between $H$. suis growth and the relative abundance of fucosylated structures. $\mathbf{b}$, e Relation between $H$. suis growth and acidic glycans. c, $\mathbf{f}$ Relation between $H$. suis growth and mucin glycans with terminal galactose. a-c The data points represent values of four mucin source per individual. d-f The data points represent the average values of four mucin source per individual. Statistics: Pearson's correlation test

In conclusion, we showed that experimental H. suis infection changed the mucin composition and glycosylation. These changes decreased the amount of $H$. suis-binding glycan structures in the pig gastric mucus niche and decreased the $H$. suis growth regulating effects of the mucins. Similarly, the $H$. suis-binding and growth-inhibiting ability of mucins from H. pylori-infected human stomachs was lower than those from noninfected individuals. Together with the observation that gastric mucin production is impaired during $H$. pylori infection, ${ }^{35}$ this demonstrates that Helicobacter spp. infections inhibit three aspects of the mucinbased defense. Inhibition of these mucus-based defenses creates a more stable and inhabitable niche, likely of importance for longterm colonization and outcome of infection. Helicobacter spp. is a group of pathogens that chronically colonize the gastric mucosa of half the world's population, cause morbidity and mortality, require severe antibiotic treatment to eradicate, and are prone to developing antibiotic resistance. Eradicating Helicobacter spp. is beneficial from a cancer prevention perspective, ${ }^{46}$ but considering the increasing problems with antibiotic-resistant bacteria, it is not justified to treat all infected people with antibiotics. Therapeutics reversing the impairments of the mucin-based defenses may provide an alternative avenue to explore in order to treat infections.

\section{METHODS}

H. suis culture conditions

H. suis strain HS1 was cultured in biphasic Brucella culture plates (BD BBL) containing 20\% fetal bovine serum (FBS) (HyClone), Vitox supplement (Oxoid), Skirrow Campylobacter selective supplement 
(Oxoid), and $5 \mathrm{mg} / \mathrm{L}$ amphotericin $\mathrm{B}$. The $\mathrm{pH}$ of the agar was adjusted to 5 with $37 \% \mathrm{HCl}$. One $\mathrm{mL}$ sterile Brucella broth with $\mathrm{pH}$ 5 was added onto the agar surface. The bacteria were cultured under microaerobic conditions $\left(10 \% \mathrm{CO}_{2}, 5 \% \mathrm{O}_{2}\right)$ at $37^{\circ} \mathrm{C}$ for 24-42 h.

\section{Samples}

Porcine. A total of 32 medicated, early weaned piglets (Rattlerow Seghers sow $x$ Piétrain boar) were used (EC approval number EC2012/129). The protocol used has previously been shown effective in limiting $H$. suis infection. ${ }^{4}$ Immediately after birth (day 0 ) as well as on day 1 and 2, the piglets received $75 \mathrm{mg}$ of amoxicillin intramuscularly (IM) (Duphamox LA ${ }^{\mathrm{TM}}$, Pfizer, USA). On day 3 , the piglets received $2.5 \mathrm{mg} / \mathrm{kg}$ of tulathromycin IM (Draxxin, Pfizer). From day 3 to day 11, all animals received $150 \mathrm{mg}$ of amoxicillin IM. On day 9, the piglets were weaned and transferred to barrier maintained facilities at the Faculty of Veterinary Medicine, Ghent University. They received a milk replacement diet using a commercial liquid feeder until the age of 35 days. At 4 weeks of age, 16 piglets were orally inoculated with $5 \mathrm{~mL}$ of Brucella broth (Becton Dickinson, Erembodegem, Belgium) with a $\mathrm{pH}$ of 5 , containing $5 \times 10^{8}$ viable $H$. suis (strain $\mathrm{HS} 1$ ) per $\mathrm{mL}$. The 16 other piglets were sham-inoculated with $5 \mathrm{~mL}$ of sterile Brucella broth with a $\mathrm{pH}$ of 5 . All pigs were euthanized 4 weeks after the experimental infection/sham administration. The Helicobacter infection status of the pig stomachs was determined by FISH on gastric histology samples and PCR on gastric mucus scrapings. ${ }^{2,4}$ Only two out of 16 sham-inoculated pigs were deemed $H$. suis free by both PCR and FISH, indicating that the medicated early weaning protocol was not entirely successful. To increase the sample number in the negative control group, we located another

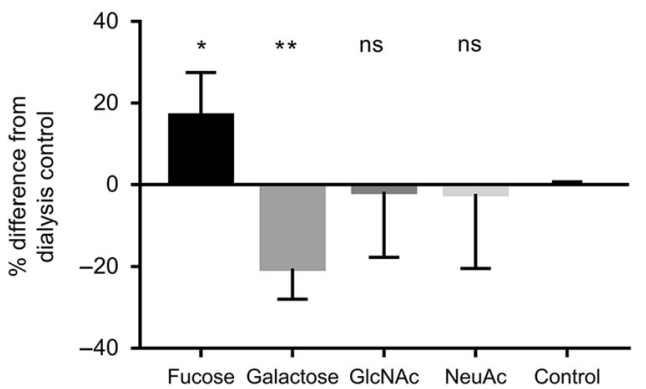

Fig. 8 Effect of monosaccharides on the $H$. suis growth. $H$. suis growth in the presence of monosaccharides $(50 \mu \mathrm{g} / \mathrm{ml})$. The values are presented as percentage growth difference from parallel cultures without added monosaccharides. Statistics: ${ }^{*} p<0.05,{ }^{* *} p$ $<0.01$, ns $p>0.05$, one-way ANOVA, Dunnett's multiple comparisons test. The data are plotted as mean \pm SEM 4-month-old $H$. suis-free pig from a different cohort. We tried to further increase the number of noninfected controls by collecting stomachs from slaughterhouses, but PCR performed on stomachs from 60 pigs determined only one free of $H$. suis, and even this pig showed signs of severe inflammation and had to be excluded from further experiments. In the $H$. suis inoculated group, all the pigs were determined $H$. suis positive with both FISH and PCR. However, multilocus sequence typing ${ }^{47}$ revealed the presence of a different strain of $H$. suis in addition to the strain used for experimental infection in 5 out of 16 pigs. Among the four pig samples included in the current study, three carried only the inoculated strain, whereas the fourth pig at a late stage was discovered to carry both the inoculated strain and another strain. Since the glycan profile and histology from this sample was similar to the other three samples, we did not exclude data from this pig.

Human. Human gastric macroscopically normal samples were obtained from obese patients in conjunction with vertical sleeve gastrectomy after giving written informed consent (Ersta Hospital, Sweden). The study protocol was approved by the regional ethics board (Regionala etikprövningsnämnden i Göteborg, Dnr 753-14). We randomly selected six Helicobacter spp. positive (gastritis scores 1-4, age 38-51, 5 females, 1 male) and seven Helicobacter spp. negative samples as controls (gastritis scores $0-1$, age 28-60, 5 females, 2 males).

Histology methods

$A B / P A S, H \& E$, and FISH staining were performed as previously described. ${ }^{32,35}$ The level of inflammation was scored blinded on $\mathrm{H} \& \mathrm{E}-$ stained sections. On pig tissue sections, inflammation scores were graded from 0 to 3 (0: no inflammatory cells or follicle, 1: moderate level of cell infiltration, no follicle, 2: widespread cell infiltration and one or two follicles and 3: widespread cell infiltration and multiple large follicles). On human tissue sections, inflammation scores graded from 0 to 5 (0: no inflammatory cells or follicle, 1: moderate level of cell infiltration, no follicle, 2: moderate level of cell infiltration one or two small follicles, 3: widespread cell infiltration and one or two small follicles 4: widespread cell infiltration and multiple follicles 5: widespread cell infiltration and multiple large follicles).

PCR for detection of Helicobacter spp. in human gastric samples Cultured $H$. pylori and a strongly positive patient sample were used as positive controls. The DNA from the samples and controls was purified with the QIAamp DNA Mini (Qiagen) kit according to the manufacturer's instructions. Helicobacter genus (16S) and $H$. pylori specific (ureC, glmM, and hspA) primers (Table 2) weresynthesized by Eurofins Genomics. The PCR was performed in $20 \mu \mathrm{L}$ of reaction volumes consisting of a final concentration of $1 \mathrm{x}$ Invitrogen ${ }^{\mathrm{TM}}$ Platinum $^{\mathrm{TM}}$ Hot Start PCR Master Mix $(2 \times$, Thermo

\begin{tabular}{|lll|}
\hline Table 2. & Primers used for PCR & \\
\hline Gene & Primer sequence $\left(5^{\prime}->3^{\prime}\right)$ & Product size bp \\
\hline Hcom 1 & Forward- GTA AAG GCT CAC CAA GGC TAT & 389 \\
Hcom 2 & Reverse - CCA CCT ACC TCT CCC ACA CTC & \\
ureC & Forward- AAG CTT TTA GGG GTG TTA GGG GTT T & 294 \\
& Reverse - CTG CTT GCT TTC TAA CAC TAA CGC & \\
glmM & Forward- TCT AAA AAC GCC CTT TCT TCT CA & 130 \\
& Reverse - ATT CGC TCA CAA ACT TAT CCC C \\
hspA & Forward- ACA GCA AGA TTC ATG CTC TT & 134 \\
& Reverse - CAG AAA TCG TTT TAG ACG GCA & 50 \\
\hline Although the ureC and the glmM primers likely are the same protein, we used this terminology to be consistent with previous literature
\end{tabular}


Fisher Scientific), $0.5 \mathrm{pmol} / \mu \mathrm{L}$ forward and reverse primer, and 5 $\mathrm{ng} / \mu \mathrm{L}$ template. Samples were run in triplicates together with positive and negative controls. The amplification program consisted of $94^{\circ} \mathrm{C}$ for $5 \mathrm{~min}, 35$ cycles $\left(94^{\circ} \mathrm{C}\right.$ for $30 \mathrm{~s}$, annealing at $60^{\circ} \mathrm{C}\left(\mathrm{g} / \mathrm{mM}\right.$, ureC, Hcom), $55^{\circ} \mathrm{C}(\mathrm{hspA})$ for $30 \mathrm{~s}$, extension at $72{ }^{\circ} \mathrm{C}$ for $30 \mathrm{~s}$ ) followed by a final extension at $72^{\circ} \mathrm{C}$ for $7 \mathrm{~min}$. Amplification was carried out using an Applied Biosystems 2720 Thermal Cycler (Thermo Fisher Scientific). The PCR products were separated using a $2 \%$ agarose gel, stained with ethidium bromide, and visualized under UV light.

\section{Isolation and characterization of gastric mucins}

Mucins were isolated from pig and human gastric tissue from the antrum and analyzed for glycan content and MUC5AC as previously described. ${ }^{18}$ Briefly, the surface and glandular mucus were separated by gentle and harsh scraping of the tissue with a glass microscope slide. Mucins were extracted from the scrapings in five volumes of extraction buffer $(6 \mathrm{M} \mathrm{GuHCl}, 5 \mathrm{mM}$ EDTA, $10 \mathrm{mM}$ sodium phosphate buffer, $\mathrm{pH} 6.5$ ) containing $0.1 \mathrm{M}$ PMSF and dispersed with Dounce homogenizer and stirred at $4{ }^{\circ} \mathrm{C}$ overnight. The insoluble material was removed by centrifugation at $23,000 \times \mathrm{g}$ for $50 \mathrm{~min}$ at $4{ }^{\circ} \mathrm{C}$ (Beckman JA-30 rotor). The pellet was reextracted twice with $10 \mathrm{~mL}$ of extraction buffer and the pooled supernatants corresponded to the $\mathrm{GuHCl}$-soluble mucins. The final pellet was reduced with $10 \mathrm{~mL}$ of $10 \mathrm{mM} 1,4-$ dithiothreitol (DTT) in reduction buffer $(6 \mathrm{M} \mathrm{GuHCl}, 5 \mathrm{mM}$ EDTA, $0.1 \mathrm{M}$ Tris- $\mathrm{HCl}$ buffer, $\mathrm{pH}$ 8.0) for $5 \mathrm{~h}$ at $37^{\circ} \mathrm{C}$ followed by alkylation with $25 \mathrm{mM}$ iodoacetamide overnight in the dark at room temperature. The suspension was centrifuged as above. The supernatant corresponded to $\mathrm{GuHCl}$-insoluble mucins and the pellet was discarded. Both the $\mathrm{GuHCl}$-soluble and $\mathrm{GuHCl}$-insoluble materials were dialyzed three times against extraction buffer using a dialysis tube with $14 \mathrm{kDa}$ pore size (Sigma-Aldrich). The mucins were isolated by isopycnic density-gradient centrifugation in $\mathrm{CsCl}$ / $4 \mathrm{M} \mathrm{GuHCl}$, as previously described. ${ }^{18}$ The density-gradient fractions were analyzed for DNA content by UV light absorbance at $280 \mathrm{~nm}$, and the density of the fractions was measured using a Carlsberg pipette as a pycnometer. The use of chaotropic agent ( $\mathrm{GuHCl}$ ) disassociates noncovalent bonds and the density-gradient centrifugation separates less glycosylated proteins/peptides from the mucin-containing fractions. ${ }^{18}$ Some of the pig gastric mucin samples were contaminated with DNA, therefore a second density-gradient centrifugation was carried out on all pig gastric samples to further purify mucins from DNA in $\mathrm{CsCl} / 0.5 \mathrm{M} \mathrm{GuHCl}{ }^{18}$ The mucin-containing fractions were then pooled into four samples (surface soluble, surface insoluble, gland soluble, and gland insoluble) from each individual.

Mass spectrometric analysis

Proteomic analysis was performed as previously described. ${ }^{18}$ Peptide MS/MS spectra were searched against UniProt and NCBI pig protein databases using Mascot server (v.2.2.04, Matrix Science Inc., MA, USA) with an in-house built mucin database. The final sequences of S. scrofa Muc5ac and Muc5b used in this project are not entirely complete and are not guaranteed to be correct in every amino acid position, but nevertheless provided the ability to efficiently discriminate between MUC5AC and MUC5B. ${ }^{18}$ For S. scrofa MUC2 and MUC6, we used protein sequences available in Genbank, XP_013845199.1 (MUC2) and XP_013845183.1 (MUC6). Both of these entries seem to comprise near full-length mucin sequences. Only peptides with a mass deviation lower than $10 \mathrm{ppm}$ were accepted, and at least two peptide sequences with manual inspection were used for positive protein identification.

Sample preparation for LC-MS/MS analysis of glycans: approx. $20 \mu \mathrm{g}$ of purified porcine gastric mucins were dot-blotted onto the polyvinylidene fluoride (PVDF) membrane (Immobilin P, Millipore, Billerica, MA, United States), stained with Alcian Blue 8GX Alcian
Blue 8GX (Sigma-Aldrich) for $1 \mathrm{~h}$ at RT and destained with methanol. The O-glycans were released and analyzed by LC-MS as described previously. ${ }^{18}$

\section{Binding assay}

Biotinylation of bacteria was performed as previously described. ${ }^{48}$ We have previously shown that binding is similar with biotinylated and un-biotinylated $H$. suis, ${ }^{18}$ although the assay with biotinylated bacteria have a lower and more consistent background signal. Mucin samples were diluted to $4 \mu \mathrm{g} / \mathrm{mL}$ in $0.5 \mathrm{M} \mathrm{GuHCl}$ and coated on Nunc ${ }^{\mathrm{TM}}$ Microplate Immuno Polysorp 96-well plates (Thermo Fisher Scientific) overnight at $4{ }^{\circ} \mathrm{C}$. The plates were washed three times with washing buffer (PBS with $0.05 \%$ Tween 20 ), and the wells were blocked for $1 \mathrm{~h}$ with $1 \%$ blocking reagent for ELISA (Roche). After discarding the blocking buffer, biotinylated bacteria were diluted to an $\mathrm{OD}_{600}$ of 0.05 in blocking buffer ( $\mathrm{pH} 2$ or $\mathrm{pH} 7)$. The bacterial suspension was added to the plates, which then were incubated for $2 \mathrm{~h}$ at $37^{\circ} \mathrm{C}$ and on a shaker $(120 \mathrm{rpm})$. The plates were washed three times and then incubated with blocking buffer containing $1 \mu \mathrm{g} / \mathrm{mL}$ of horseradish peroxidase-conjugated streptavidin for $1 \mathrm{~h}$ at room temperature. After three further washing steps, TMB substrate was added and the plates were incubated for $15 \mathrm{~min}$ at room temperature. The reaction was stopped by adding an equivalent amount of $0.5 \mathrm{M} \mathrm{H}_{2} \mathrm{SO}_{4}$ and the plates were read in a microplate reader at $450 \mathrm{~nm}$.

$H$. suis growth assay with mucins and monosaccharides

$H$. suis growth was determined by RealTime-Glo ${ }^{\mathrm{TM}}$ MT Cell Viability Assay (Promega), in which the luminescent signal is proportional to the number of viable cells. Prior to the assay, the temperature of MT Cell Viability Substrate and NanoLuc ${ }^{\circledR}$ Enzyme and the culture medium (Brucella broth (pH 5) containing 20\% FBS) was equilibrated to $37^{\circ} \mathrm{C}$. H. suis was harvested from agar plates, centrifuged at $2500 \times \mathrm{g}$ for $3 \mathrm{~min}$, and resuspended in the culture medium. Mucin samples were dialyzed using $12 \mathrm{kDa}$ dialysis tubes (Sigma-Aldrich); four times against $2 \mathrm{M} \mathrm{NaCl}$, then two times against PBS. The mucin and monosaccharide samples were diluted in PBS. $H$. suis with a final $\mathrm{OD}_{600}$ of 0.2 was added to 96 -well white opaque-walled tissue culture plates in the presence of $50 \mu \mathrm{g} / \mathrm{mL}$ mucin or monosaccharide. Adding dialyzed mucin isolation buffer instead of mucin to the culture medium was used as a control for normal growth. For the pig mucins, the growth in response to each mucin source was analyzed, and the mean growth per pig was calculated based on these results. For the human samples, the four mucin sources were pooled before the assay. MT Cell Viability Substrate and NanoLuc ${ }^{\circledR}$ Enzyme were added to the bacterial suspension according to the manufacturer's instructions. The plates were covered with gas permeable seal (Diversified Biotech) and incubated at $37^{\circ} \mathrm{C}$ inside a plate reader (CLARIOstar, BGM labtech $\mathrm{GmbH}$, Ortenberg, Germany) for $24 \mathrm{~h}$ at $37^{\circ} \mathrm{C}$ under microaerobic conditions $\left(10 \% \mathrm{CO}_{2}, 5 \% \mathrm{O}_{2}\right)$. Luminescence reading was measured at every $30 \mathrm{~min}$.

\section{Statistical analyses}

Statistical analyses were performed using the GraphPad Prism 7 software. Mann-Whitney $U$ tests were used to analyze differences between infected and noninfected groups, where the data were presented per individual. D'Agostino-Pearson omnibus normality tests were performed prior to performing analyses of variance (ANOVA), and the datasets passed the tests. Two-way ANOVAs were performed to analyze differences between infected and noninfected groups, where the data were presented per mucin source. Differences between more than two groups and a single control group were analyzed by one-way ANOVA followed by Dunnett's multiple comparisons test. Relations between parameters were analyzed by Pearson's correlation test. Differences with $p \leq 0.05$ were considered significant. 


\section{ACKNOWLEDGEMENTS}

This work was supported by the Swedish research council, the Swedish research council Formas (221-2011-1036 and 221-2013-590), the Swedish Cancer Society, the Ragnar Söderberg, RR Julin, Jeansson WM Lundgren and Erling-Persson Family Foundations, the European Union FP7 GastricGlycoExplorer ITN (316929) and the Research Fund of Ghent University (Grant no. GOA01G0040 and BOF14/GOA/010). The funders had no role in study design, data collection and analysis, decision to publish, or preparation of the paper.

\section{AUTHOR CONTRIBUTIONS}

M.P., S.K.L., and F.H. designed the project and wrote the paper. M.P., B.A., C.J., M.E., G. C., B.F., A.S., and R.D. performed experiments, M.P., B.A., C.J., M.E., G.C., N.G.K., and S.K. L. analyzed data, A.T. provided human gastric samples, F.H. provided the bacterial strain. All authors reviewed the paper.

\section{ADDITIONAL INFORMATION}

The online version of this article (https://doi.org/10.1038/s41385-019-0154-4) contains supplementary material, which is available to authorized users.

Competing interests: The authors declare no competing interests.

\section{REFERENCES}

1. Barbosa, A. J., Silva, J. C., Nogueira, A. M., Paulino Junior, E. \& Miranda, C. R. Higher incidence of Gastrospirillum sp. in swine with gastric ulcer of the pars oesophagea. Vet. Pathol. 32, 134-139 (1995).

2. De Groote, D. et al. Detection of "Candidatus Helicobacter suis" in gastric samples of pigs by PCR: comparison with other invasive diagnostic techniques. J. Clin. Microbiol. 38, 1131-1135 (2000)

3. De Witte, C. et al. Helicobacter suis induces changes in gastric inflammation and acid secretion markers in pigs of different ages. Vet. Res. 48, 34 (2017).

4. De Bruyne, E. et al. An experimental Helicobacter suis infection causes gastritis and reduced daily weight gain in pigs. Vet. Microbiol. 160, 449-454 (2012).

5. Queiroz, D. M. et al. Association between Helicobacter and gastric ulcer disease of the pars esophagea in swine. Gastroenterology 111, 19-27 (1996).

6. Van den Bulck, K. et al. Identification of non-Helicobacter pylori spiral organisms in gastric samples from humans, dogs, and cats. J. Clin. Microbiol. 43, 2256-2260 (2005).

7. Debongnie, J. C., Donnay, M. \& Mairesse, J. Gastrospirillum hominis ("Helicobacter heilmanii"): a cause of gastritis, sometimes transient, better diagnosed by touch cytology? Am. J. Gastroenterol. 90, 411-416 (1995).

8. Debongnie, J. C. et al. Gastric ulcers and Helicobacter heilmannii. European journa of gastroenterology \&. Hepatology 10, 251-254 (1998).

9. Morgner, A., Bayerdorffer, E., Meining, A., Stolte, M. \& Kroher, G. Helicobacter heilmannii and gastric cancer. Lancet 346, 511-512 (1995).

10. Haesebrouck, F. et al. Gastric helicobacters in domestic animals and nonhuman primates and their significance for human health. Clin. Microbiol. Rev. 22, 202-223 (2009).

11. Stolte, M. et al. A comparison of Helicobacter pylori and $H$. heilmannii gastritis. A matched control study involving 404 patients. Scand. J. Gastroenterol. 32, 28-33 (1997).

12. Nakamura, M. et al. "Candidatus Helicobacter heilmannii" from a cynomolgus monkey induces gastric mucosa-associated lymphoid tissue lymphomas in C57BL/6 mice. Infect. Immun. 75, 1214-1222 (2007).

13. O'Rourke, J. L., Dixon, M. F., Jack, A., Enno, A. \& Lee, A. Gastric B-cell mucosaassociated lymphoid tissue (MALT) lymphoma in an animal model of 'Helicobacter heilmannii' infection. J. Pathol. 203, 896-903 (2004).

14. Quintana-Hayashi, M. P., Padra, M., Padra, J. T., Benktander, J. \& Linden, S. K. Mucus-pathogen interactions in the gastrointestinal tract of farmed animals. Microorganisms 6, E55 (2018).

15. McGuckin, M. A., Linden, S. K., Sutton, P. \& Florin, T. H. Mucin dynamics and enteric pathogens. Nat. Rev. Microbiol. 9, 265-278 (2011).

16. Buisine, M. P. et al. Developmental mucin gene expression in the gastroduodenal tract and accessory digestive glands. I. Stomach. A relationship to gastric carcinoma. J. Histochem. Cytochem.: Off. J. Histochem. Soc. 48, 1657-1666 (2000).

17. Reis, C. A. et al. Immunohistochemical study of the expression of MUC6 mucin and co-expression of other secreted mucins (MUC5AC and MUC2) in human gastric carcinomas. J. Histochem. Cytochem.: Off. J. Histochem. Soc. 48, 377-388 (2000).

18. Padra, M. et al. Helicobacter suis binding to carbohydrates on human and porcine gastric mucins and glycolipids occurs via two modes. Virulence 9, 1-48 (2018)
19. Robbe, C., Capon, C., Coddeville, B. \& Michalski, J. C. Structural diversity and specific distribution of O-glycans in normal human mucins along the intestinal tract. Biochem. J. 384(Pt 2), 307-316 (2004).

20. Linden, S. K., Sutton, P., Karlsson, N. G., Korolik, V. \& McGuckin, M. A. Mucins in the mucosal barrier to infection. Mucosal Immunol. 1, 183-197 (2008).

21. Padra, J. T. et al. Aeromonas salmonicida growth in response to atlantic salmon mucins differs between epithelial sites, is governed by sialylated and $\mathrm{N}$ acetylhexosamine-containing O-glycans, and is affected by $\mathrm{Ca}(2)$. Infect. Immun. 85, e00189-17 (2017)

22. Frenkel, E. S. \& Ribbeck, K. Salivary mucins protect surfaces from colonization by cariogenic bacteria. Appl. Environ. Microbiol. 81, 332-338 (2015).

23. Miller, R. S. \& Hoskins, L. C. Mucin degradation in human colon ecosystems. Fecal Popul. densities mucin-degrading Bact. Estim. a "most Probable number" Method. Gastroenterol. 81, 759-765 (1981).

24. Martens, E. C., Chiang, H. C. \& Gordon, J. I. Mucosal glycan foraging enhances fitness and transmission of a saccharolytic human gut bacterial symbiont. Cell. Host. Microbe 4, 447-457 (2008).

25. Schreiber, S. et al. The spatial orientation of Helicobacter pylori in the gastric mucus. Proc. Natl Acad. Sci. USA 101, 5024-5029 (2004).

26. Linden, $S$. et al. Role of $A B O$ secretor status in mucosal innate immunity and $H$. pylori infection. PLoS Pathog. 4, e2 (2008).

27. Linden, S., Semino-Mora, C., Liu, H., Rick, J. \& Dubois, A. Role of mucin Lewis status in resistance to Helicobacter pylori infection in pediatric patients. Helicobacter 15 251-258 (2010).

28. Linden, S. K. et al. MUC1 limits Helicobacter pylori infection both by steric hindrance and by acting as a releasable decoy. PLoS Pathog. 5, e1000617 (2009).

29. Skoog, E. C. et al. Human gastric mucins differently regulate Helicobacter pylori proliferation, gene expression and interactions with host cells. PLOS. ONE. 7 e36378 (2012).

30. Skoog, E. C. et al. BabA dependent binding of Helicobacter pylori to human gastric mucins cause aggregation that inhibits proliferation and is regulated via ArsS. Sci. Rep. 7, 40656 (2017).

31. Freitas, M., Axelsson, L. G., Cayuela, C., Midtvedt, T. \& Trugnan, G. Microbial-host interactions specifically control the glycosylation pattern in intestinal mouse mucosa. Histochem. Cell. Biol. 118, 149-161 (2002).

32. Jin, C. et al. Atlantic salmon carries a range of novel O-glycan structures differentially localized on skin and intestinal mucins. J. Proteome Res. 14, 3239-3251 (2015).

33. Venkatakrishnan, V. et al. Brachyspira hyodysenteriae infection regulates mucin glycosylation synthesis inducing an increased expression of core-2 O-glycans in Porcine colon. J. Proteome Res. 16, 1728-1742 (2017).

34. Byrd, J. C., Yan, P., Yunker, C. \& Bresalier, R. S. Aberrant expression of gland-type gastric mucin in the surface epithelium of $\mathrm{H}$-pylori-infected patients. Gastroenterology 112, A80-A80 (1997).

35. Navabi, N., Johansson, M. E., Raghavan, S. \& Linden, S. K. Helicobacter pylori infection impairs the mucin production rate and turnover in the murine gastric mucosa. Infect. Immun. 81, 829-837 (2013).

36. Kawakubo, M. et al. Natural antibiotic function of a human gastric mucin against Helicobacter pylori infection. Sci. (New York, NY) 305, 1003-1006 (2004).

37. Kocer, B. et al. A confirmatory report for the close interaction of Helicobacter pylori with gastric epithelial MUC5AC expression. J. Clin. Gastroenterol. 38, 496-502 (2004).

38. Quintana-Hayashi, M. P. et al. BabA-mediated adherence of pediatric ulcerogenic H. pylori strains to gastric mucins at neutral and acidic pH. Virulence 9, 1699-1717 (2018).

39. Mahdavi, J. et al. Helicobacter pylori SabA adhesin in persistent infection and chronic inflammation. Science 297, 573-578 (2002).

40. Linden, S. K., Wickstrom, C., Lindell, G., Gilshenan, K. \& Carlstedt, I. Four modes of adhesion are used during Helicobacter pylori binding to human mucins in the oral and gastric niches. Helicobacter 13, 81-93 (2008).

41. Mcguckin, M. A. et al. Muc1 mucin limits both Helicobacter pylori colonization of the murine gastric mucosa and associated gastritis. Gastroenterology 133 1210-1218 (2007).

42. Morales-Espinosa, R. et al. Colonization of Mexican patients by multiple Helicobacter pylori strains with different vacA and cagA genotypes. J. Clin. Microbiol. 37, 3001-3004 (1999).

43. Boyanova, L. et al. High prevalence of virulent Helicobacter pylori strains in symptomatic Bulgarian patients. Diagn. Microbiol. Infect. Dis. 64, 374-380 (2009).

44. Liu, J. et al. Prevalence of coinfection with gastric non-Helicobacter pylori Helicobacter (NHPH) species in Helicobacter pylori-infected patients suffering from gastric disease in Beijing, China. Helicobacter 20, 284-290 (2015).

45. Peng, $X$. et al. Non-pylori Helicobacters (NHPHs) induce shifts in gastric microbiota in Helicobacter pylori-infected patients. Front. Microbiol. 8, 1038 (2017) 
46. Doorakkers, E., Lagergren, J., Engstrand, L. \& Brusselaers, N. Eradication of Helicobacter pylori and gastric cancer: a systematic review and meta-analysis of cohort studies. J. Natl Cancer Inst. 108, djw132 (2016).

47. Liang, J. et al. Multilocus sequence typing of the porcine and human gastric pathogen Helicobacter suis. J. Clin. Microbiol. 51, 920-926 (2013).

48. Lindén, S. et al. Strain- and blood group-dependent binding of Helicobacter pylori to human gastric MUC5AC glycoforms. Gastroenterology 123, 1923-1930 (2002).

49. Choi, Y. K., Han, J. H. \& Joo, H. S. Identification of novel Helicobacter species in pig stomachs by PCR and partial sequencing. J. Clin. Microbiol. 39, 3311-3315 (2001).

50. Puz, S. et al. A novel noninvasive genotyping method of Helicobacter pylori using stool specimens. Gastroenterology 135, 1543-1551 (2008).

51. Dolan, B. et al. The use of stool specimens reveals Helicobacter pylori strain diversity in a cohort of adolescents and their family members in a developed country. Int. J. Med. Microbiol. 308, 247-255 (2018).
Open Access This article is licensed under a Creative Commons Attribution 4.0 International License, which permits use, sharing, adaptation, distribution and reproduction in any medium or format, as long as you give appropriate credit to the original author(s) and the source, provide a link to the Creative Commons license, and indicate if changes were made. The images or other third party material in this article are included in the article's Creative Commons license, unless indicated otherwise in a credit line to the material. If material is not included in the article's Creative Commons license and your intended use is not permitted by statutory regulation or exceeds the permitted use, you will need to obtain permission directly from the copyright holder. To view a copy of this license, visit http://creativecommons. org/licenses/by/4.0/.

(c) The Author(s) 2019 